\title{
Medication nonadherence and psychiatry
}

\author{
Sarah C.E. Chapman and Rob Horne
}

\begin{abstract}
Purpose of review
Nonadherence to appropriately prescribed medication for psychiatric disorders prevents patients from realizing the full benefits of their treatment and negatively impacts on individuals, their families and the healthcare system. Understanding and reducing nonadherence is therefore a key challenge to quality care for patients with psychiatric disorders. This review highlights findings regarding the prevalence and consequence of nonadherence, barriers to adherence and new intervention methods from 2012 onwards.
\end{abstract}

\section{Recent findings}

Recent research has highlighted that nonadherence is a global challenge for psychiatry and has linked nonadherence to poorer outcomes, including hospital admissions, suicide and mortality. Optimizing medication regimens can reduce nonadherence; however, often a complex interplay of factors affects individuals' motivation and ability to follow their prescription. Psychiatrists can enable patients to develop an accurate model of their illness and treatment and facilitate adherence. However, nonadherence is often a hidden issue within consultations. Novel interventions using new technologies and tailoring techniques may have the potential to reduce nonadherence.

\section{Summary}

Nonadherence remains a significant challenge for patients with psychiatric disorders, physicians and healthcare systems. New developments demonstrate the importance of developing tailored interventions to enable patients to overcome perceptual and practical barriers to adherence.

\section{Keywords}

bipolar disorder, intervention, medication adherence, psychiatry, schizophrenia

\section{INTRODUCTION}

Taking the prescribed dose of medication, at the correct time, and for the full course of treatment is fundamental to patients realizing the full potential benefits of medications. However, between 30 and $50 \%$ of medicines for long-term conditions are not taken as prescribed, resulting in costs for individual patients and healthcare systems [1]. Research suggests that rates of nonadherence in patients with psychiatric disorders are comparable to those of patients with other long-term conditions $[2,3]$.

The Perceptions and Practicalities Approach (PaPA) has been used to describe factors contributing to nonadherence in psychiatric disorders $[4,5]$ (see Fig. 1). This states that nonadherence is the product of a range of perceptual factors (e.g. patients' beliefs about their illness and treatment) and practical factors (e.g. capability and resources) influencing their motivation and ability to start and continue with treatment. Specifically, the model emphasizes that, even when patients have the ability and opportunity to take their medication as prescribed, they may lack the motivation to adhere. Thus, to understand and address nonadherence, we need to view the illness and the treatment from the patients' perspective. Within this approach, two key beliefs have been implicated in the way in which medications are prescribed: belief in personal need for medication and concerns about the potential adverse consequences of medication. This Necessity-Concerns Framework has been found to have utility in explaining nonadherence across long-term conditions, including within psychiatric disorders [6].

Effective interventions to improve adherence remain elusive; a recent Cochrane review has called for more innovative interventions $[7,8]$. To

Centre for Behavioural Medicine, UCL School of Pharmacy, London, UK Correspondence to Rob Horne, Centre for Behavioural Medicine, Department of Practice and Policy, UCL School of Pharmacy, Entrance A, BMA House, Tavistock Square, London WC1H 9JP, UK. Tel: +44 020 7874 1281; fax: +44 0207387 5693; e-mail: r.horne@ucl.ac.uk

Curr Opin Psychiatry 2013, 26:446-452

DOI:10.1097/YCO.0b013e3283642da4

This is an open access article distributed under the Creative Commons Attribution License 4.0, which permits unrestricted use, distribution, and reproduction in any medium, provided the original work is properly cited. 


\section{KEY POINTS}

- Nonadherence remains a global challenge for psychiatry that has been linked to suicide rates, all-cause mortality and hospitalization for patients.

- Adherence to psychiatric medications is a complex, dynamic behaviour requiring patients to initiate treatment and continue to take their medications at the correct time, in the correct dose, for prolonged periods of time.

- Modifying practical barriers to adherence (e.g. dose frequency) can reduce nonadherence; however, patients also need to be motivated to adhere by an informed understanding of their illness and treatment.

- A strong alliance between patients and psychiatrists can protect against nonadherence in part by enabling patients to develop an accurate model of their illness and treatment.

- Effective adherence interventions remain elusive; however, new methods of tailoring content to the needs of individual patients combined with the use of technologies such as electronic monitoring and SMS may prove effective.

achieve this, we need to apply sound theories of the causes of nonadherence and theories for changing medication-taking behaviour, the why and how of nonadherence [5]. This review will therefore focus on recent developments from 2011 in the field of nonadherence within psychiatric disorders, with particular focus on the determinants of adherence and interventions to promote adherence.

\section{NONADHERENCE IS A GLOBAL CHALLENGE FOR PSYCHIATRY}

Nonadherence to psychiatric medication is a global problem. Recent research has highlighted nonadherence as a key challenge to psychiatric care in

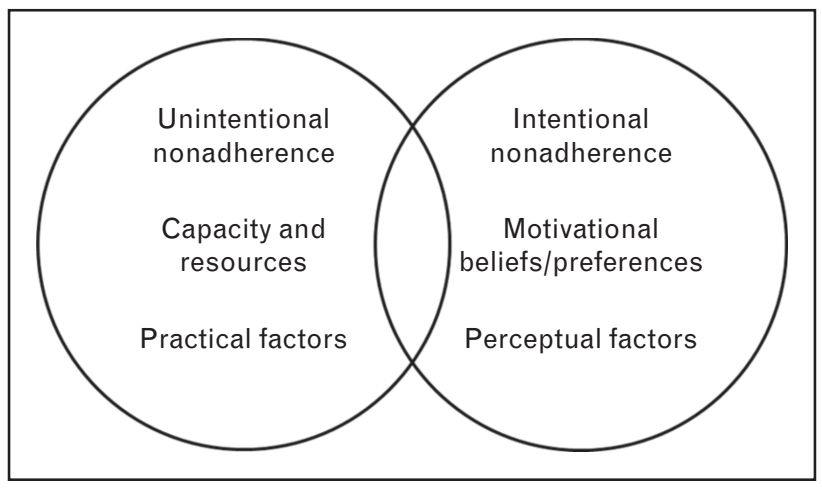

FIGURE 1. Perceptions and Practicalities Approach to nonadherence [5].
Asia [9-11], Europe [12-14,15"'], South America [16], North America [17",18-20,21"], Australia [22], the Middle East [23"] and Africa [24"]. In Africa, Alene et al. [24"] evaluated adherence rates to antipsychotic medication in patients receiving care at an Ethiopian hospital. They found that, although $52.1 \%$ reported that they had never missed a dose or taken it outside the time instructed, only 19.6\% were fully adherent if assessed using pharmacy refill records, illustrating that nonadherence can be underestimated in this setting, similarly to discrepancies shown in other settings, for example in a South Korean sample of outpatients with schizophrenia [25].

\section{PATTERNS OF NONADHERENCE IN PSYCHIATRIC DISORDERS}

Realizing the full benefits of an appropriately prescribed medication is conditional on optimal adherence. However, in practice, adherence is a complex, dynamic behaviour; patients can be nonadherent to their prescribed regimen because they do not initiate, accurately maintain or persist with their regimen. A new taxonomy of adherence terminology has been developed to promote consistency in the terminology used to describe adherence [ $\left.26^{-"}\right]$. This describes adherence as being composed of initiation (taking the first dose of the medication), discontinuation (no longer taking the medication) and implementation (whether the patient's actual behaviour corresponds to the prescribed dosing regimen in the period from initiation to discontinuation). Within this taxonomy, persistence refers to the time between initiation and the final dose of the medication. Implementation nonadherence can include a range of differences between the actual regimen execution and that prescribed, including missing doses, taking a different dose of medication than that prescribed and taking medication at different times.

Different assessment methods can be used to identify these differing types of nonadherence; for example, Yang et al. [25] used pill counts to assess whether the patient had taken the correct number of doses, and electronic monitoring to assess whether patients had taken these doses on schedule. Other researchers have used nonpersistence with a regimen to assess nonadherence, often using pharmacy refill (e.g. $\left[21^{\prime \prime}, 27\right]$ ). Through understanding the different aspects of adherence measured by these assessments, we can understand a lack of correspondence between measurements and begin to investigate whether different patterns of adherence have different causes and consequences. 
Recent research has highlighted different patterns of nonadherence. Jaeger et al. [28] used latent class analysis to group 371 inpatients with schizophrenia on the basis of their scores on the Medication Adherence Rating Scale at baseline, a self-report/clinician-report tool. They identified five groups of patients on the basis of their adherence responses and found that different patterns of adherence were associated with different rates of later discontinuation and hospitalization. Chang et al. [9] categorized schizophrenic patients on the basis of type of nonadherence as measured by pharmacy refill and found that different patterns of nonadherence were associated with different antipsychotic drugs. Offord et al. [21"] found that patients with schizophrenia who had early nonadherence within 90 days of starting a new prescription had lower adherence for the following 12 months.

\section{IMPACT OF NONADHERENCE}

Evidence for an impact of nonadherence on outcomes for psychiatric patients has been consolidated within new research. Nonadherence has been found to predict poorer outcomes for patients, including hospital admission [29,30], violence [31"'], suicide and premature mortality. San et al. [30] analysed the medical records of patients with schizophrenia/schizoaffective disorder admitted to acute care. Of the 1646 patients on whom data were available, for $58.6 \%$ of patients the main reason for hospital admission was listed as nonadherence.

Nonadherence can also be a risk factor for violence in psychosis, as confirmed by a recent meta-analysis [31"']. Witt et al. [31"'] found a moderate association between violence (including a range of measures such as reported aggression and arrests) and adherence to medication [odds ratio (OR) 2.0] on the basis of nine studies.

In suicide research, adherence has been identified as the 'strongest modifiable protective factor' against suicide in patients with bipolar disorder who were followed for 10 years [32]. Lindstrom et al. [12] analysed the autopsy blood samples of 33 patients from a psychosis clinic who had committed suicide over a 7-year period. They found that plasma drug levels for prescribed antipsychotic and antidepressant medications suggested that three out of 24 were nonadherent to antipsychotics and 10 out of 10 were nonadherent to antidepressants at the time of their suicide. Similarly, Ruengorn et al. [33"] used a case-control methodology and estimated the odds of suicide attempts in patients with major depressive disorder as approximately twice as high for patients with low adherence.
Nonadherence has also been associated with premature mortality in schizophrenia [34"]. Cullen et al. [34"] retrospectively examined the records of a cohort of US patients with schizophrenia and found that low adherence to antipsychotics predicted mortality. As with previous research showing an association between mortality and adherence [35], it is unclear whether adherence per se is contributing to reduced mortality, or whether it is a marker for other factors. For example, as stated above, adherence could have a direct effect on psychiatric outcomes and associated risk. However, adherence may be a marker for a more direct causal effect, for example good social support or a tendency to engage in health behaviours, including exercise.

\section{NONADHERENCE IS OFTEN A HIDDEN PROBLEM}

It is very difficult for practitioners to guess which of their patients are nonadherent $\left[11,23^{-}, 36\right]$. Olivares et al. [23"] surveyed 4722 psychiatrists across 36 countries. Psychiatrists estimated that 53\% of their patients with schizophrenia were nonadherent, suggesting that they recognized the importance of nonadherence. However, identifying the particular patients who are not adherent may be a particular challenge. Stephenson et al. [37"'] asked US physicians to rate the adherence of individual patients with bipolar disorder and/or schizophrenia, and found that $72 \%$ of patients whose prescription refill records indicated nonadherence were rated as adherent by their physician. Thus, there is evidence that nonadherence may be recognized as a general problem but remains hidden within individual consultations. Patients may fear that physicians will interpret a lack of faith in their medicine as representing a lack of faith in them, and therefore be reluctant to raise their concerns and doubts with their physicians.

A strong, trusting relationship between patients and physicians may therefore protect against nonadherence, as confirmed by a new meta-analysis that found that, where the alliance between clinicians and patients was strong, patients were more likely to adhere [38"]. Thompson and McCabe [38"] identified 23 studies relevant to this topic and suggested that future studies should focus on objective assessments of the content of clinical interactions. In one study that did directly assess consultation content, Quirk et al. [39"'] analysed recordings of 92 consultations that contained discussion of antipsychotic medications between patients and nine UK psychiatrists. Nonadherence was disclosed in 22 consultations and most frequently resulted in a prescription change in 
line with the behaviour of the patient [39""]. The researchers suggest that physicians may avoid risking conflict around medication adherence in order to maintain patients' engagement with treatment. However, research on the causes of nonadherence suggests that addressing patients' doubts and concerns about medication directly may be essential to supporting adherence $[6,40,41]$.

\section{DETERMINANTS OF NONADHERENCE}

Some medication regimens appear to be associated with greater adherence due to specific features of the medication, such as side effect profiles [27] or reduced dosing frequency (as shown by recent systematic reviews) [42,43]. However, even where regimens are optimized for adherence by reducing the number or frequency of doses, motivation is still required to maintain full adherence.

Within the PaPA, adherence to medication is determined by both practical factors (e.g. resources, patient capacity) and perceptual factors (e.g. beliefs about treatment and illness) (see Fig. 1). Hence, attempts to improve adherence by tackling practical barriers alone may not be successful if perceptual barriers (e.g. doubts about medication necessity or concerns about adverse consequences) are salient for individuals. Staring et al. [44] found that a lack of insight, and in particular low recognition of the need for treatment, was strongly associated with nonadherence within a sample of patients with schizophrenia. This effect was particularly strong when patients had good verbal memory. Even where practical barriers are important, they may reinforce or overlap with perceptual barriers. For example, a cross-sectional survey of 24017 US patients with chronic illnesses, including depression, found that perceptual factors predicted reported unintentional nonadherence (e.g. forgetting, running out of medication, affordability), suggesting that motivational factors can impact on both perceptual and practical barriers to adherence [45].

The key role of medication beliefs in adherence is illustrated by a recent qualitative synthesis of research on this topic in patients with schizophrenia, which highlighted beliefs about control, dependence and stigma [46]. Richardson et al. [47] conducted a meta-analysis of the association between medication attitudes and adherence in patients with psychoses, finding 14 studies indicating a small to moderate association. A need for more prospective and intervention studies was identified. Patients with a lack of insight into their psychiatric disorder have also been found to be at risk of nonadherence [13]. These studies demonstrate that motivational factors extend beyond knowledge of medication efficacy and adverse effects.

Comorbid psychiatric conditions can themselves be risk factors for nonadherence. Depression and substance abuse have both been linked to nonadherence within patients with psychiatric diagnoses $\left[32,48^{-{ }^{*}}, 49^{\boldsymbol{\prime}}\right]$. Psychiatric comorbidity is also a risk factor for nonadherence in physical disorders. Kronish et al. [50"] found that veterans with post-traumatic stress disorder (PTSD) had increased odds of nonadherence to all medications, relative to veterans without PTSD (OR 1.47), partly due to increased concerns about the potential adverse effects of treatment [51]. Similarly, Rao et al. [52] found that nonadherence in HIV patients was predicted by symptoms of depression, which were also associated with increased perceived HIV stigma. Comorbid psychiatric conditions may therefore impact on both practical barriers to adherence (e.g. through increased regimen complexity) and perceptual barriers (e.g. through increased concerns).

\section{NEW DEVELOPMENTS IN ADHERENCE INTERVENTIONS}

Adherence interventions require three key components: content (e.g. information, feedback), delivery vehicle (e.g. face-to-face nurse support, telephone calls, e-mail) and a broader context (e.g. primary care setting, inpatient care); see Fig. 2 [5].

\section{Intervention content}

New developments have focused on intervention content, in particular tailoring, cognitive behavioural therapy (CBT) techniques and financial incentives. Sajatovic et al. $[53,54]$ found improvements in adherence in a group of patients with bipolar disorder who were screened on factors including attitudes towards their medicines and

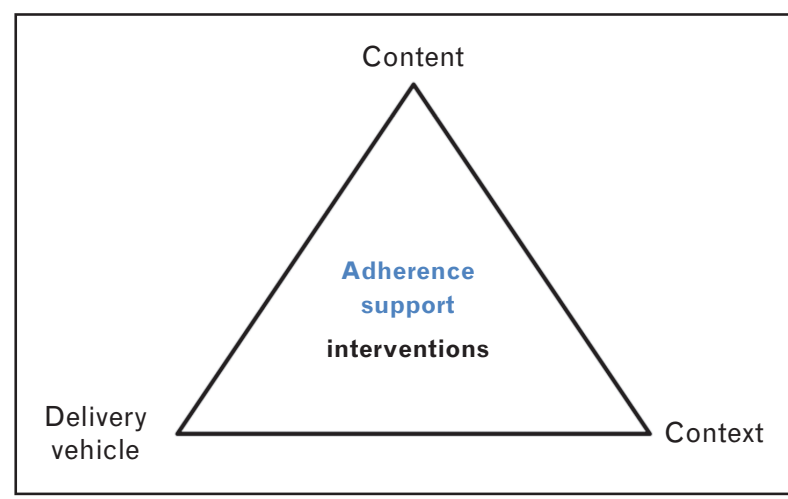

FIGURE 2. Intervention components, based on content from $[5]$. 
assigned intervention content on this basis. However, a fully controlled trial is needed before strong conclusions can be drawn regarding the utility of this tailoring process. Gleeson et al. [55] addressed medication adherence as part of relapse prevention therapy for first-episode psychosis, a CBT-based intervention composed of 7 months of fortnightly individual therapy sessions along with family intervention. They found that medication adherence improvements were sustained at 30 months of follow-up. The use of financial incentives in order to motivate adherence is one strategy that a new systematic review has indicated may be effective across long-term conditions, including psychiatric disorders [56]. However, questions remain regarding the ethics of this approach, in particular the implications for informed choice relating to treatments wherein there is potential for harm as well as benefit.

\section{Delivery vehicle}

Novel methods of delivering interventions have been applied within psychiatric settings, including computerized decision tools [57"], electronic monitoring and feedback [58"'], SMS [15"'] and telemonitoring [59]. Results from these approaches are promising, but the evidence for efficacy is mixed. Montes et al. [15"'] reported improvements in adherence for patients with schizophrenia who were sent 3 months of daily SMS reminders; however, patients were only followed for 3 months postintervention and a substantial proportion of intervention patients who did not receive all SMS messages were excluded from the sample. Velligan et al. [58"-] found that patients with schizophrenia who were given electronic pill cap reminder devices had equivalent improvements in adherence to those given $30 \mathrm{~min}$ of individualized support per week for 9 months. However, they found higher dropouts in the electronic pill cap condition and did not follow up patients postintervention. Questions therefore remain about whether these new intervention delivery vehicles are suitable for all patients, and whether they result in long-term improvements in adherence.

\section{Intervention context}

Intervention content and delivery may need to be contextualized to environmental factors, including cultural variation. For example, Read [60"] conducted qualitative interviews with patients taking antipsychotic medication in Ghana, and highlighted reasons for nonadherence, including side effects impacting on the ability to perform physical work, and a desire for a permanent 'cure' rather than a maintenance therapy. Thus, particular beliefs may be more relevant to some individuals within particular settings. However, it is important to recognize that variations in treatment perceptions and behaviours such as adherence are likely to be greater within cultural groups than across groups [61]. Hence, intervention content should be tailored to the individual rather than across a cultural group. Kopelowicz et al. [62"'] reported a culturally adapted family intervention for Mexican American patients with schizophrenia, which improved adherence at 12 months postintervention, illustrating ways in which the intervention content and delivery can be addressed to barriers specific to the patient group without relying on generalizations about which barriers patients would face on the basis of their background. For example, they tailored intervention content on the basis of the outcomes from individual interviews with patients. By personalizing intervention content based on individual attitudes, the researchers ensured culturally relevant content without making generalizations about the typical beliefs held by a specific group.

In summary, new interventions have been developed, and questions around their long-term effectiveness and relevance across diverse patient groups remain. In addition, one key challenge for adherence intervention development is to systematically describe intervention components to enable researchers to isolate the effective components of interventions and facilitate understanding and replication interventions [7]. For example, currently, both or neither of two interventions described as psychoeducational may address concerns about side effects. Within the field of behaviour change, a taxonomy to describe intervention components has been developed over recent years [63"'], which may be applicable to adherence interventions. By understanding the processes and active components within effective interventions, we may be able to open the 'black box' of intervention techniques and produce brief interventions tailored to the needs of individual patients [7].

\section{CONCLUSION}

Nonadherence remains a challenge for patients with psychiatric disorders, their physicians and healthcare providers, resulting in poorer outcomes for patients. New developments in the field of psychiatry shed light on key modifiable determinants of nonadherence within psychiatric disorders. Using this understanding to build theories of both adherence and behaviour change is a key challenge 
[5]. New technologies may also be able to support adherence within low-resource settings.

\section{Acknowledgements}

We would like to thank Lindsay Macdonald for her helpful comments on a draft of this review. No funding was available for this study.

\section{Conflicts of interest}

There are no conflicts of interest.

\section{REFERENCES AND RECOMMIENDED \\ READING}

Papers of particular interest, published within the annual period of review, have been highlighted as:

- of special interest

- of outstanding interest

Additional references related to this topic can also be found in the Current

World Literature section in this issue (pp. 000-000).

1. World Health Organization. Adherence to long-term therapies: evidence for action. Geneva, Switzerland: World Health Organization; 2003.

2. Bulloch AM, Patten S. Nonadherence with psychotropic medications in the general population. Soc Psychiatry Psychiatr Epidemiol 2010; 45: $47-56$.

3. Cramer JA, Rosenheck R. Compliance with medication regimens for mental and physical disorders. Psychiatr Serv 1998; 49:196-201.

4. Clatworthy J, Bowskill R, Rank T, et al. Adherence to medication in bipolar disorder: a qualitative study exploring the role of patients' beliefs about the condition and its treatment. Bipolar Disord 2007; 9: 656-664.

5. Horne R, Clatworthy J. Adherence to advice and treatment. In: French D, Vedhara K, Keptein AA, Weinman J, editors. Health psychology. 2nd ed. Chichester: British Psychological Society and Blackwell Publishing Ltd; 2010. pp. 175-188.

6. Clatworthy J, Bowskill R, Parham R, et al. Understanding medication nonadherence in bipolar disorders using a Necessity-Concerns Framework. J Affect Disord 2009; 116:51-55.

7. Horne R, Weinman J, Barber N, et al. Concordance, adherence and compliance in medicine taking: a conceptual map and research priorities. London: National Institute for Health Research (NIHR) Service Delivery and Organisation (SDO) Programme. 2005; <http://www.sdo.Ishtm.ac.uk/sdo762004. $\mathrm{html}>2006$. [Accessed 12 February 2013]

8. Haynes RB, Yao X, Degani A, et al. Interventions to enhance medication adherence. Cochrane Database Syst Rev 2005; CD000011.

9. Chang $\mathrm{CM}, \mathrm{Wu} \mathrm{KY}$, Liang $\mathrm{HY}$, et al. Adherence patterns with first- versus second-generation antipsychotics for newly diagnosed schizophrenia in Taiwan. Psychiatr Serv 2012; 63:504-507.

10. Wu CS, Lin YJ, Feng J. Trends in treatment of newly treated schizophreniaspectrum disorder patients in Taiwan from 1999 to 2006. Pharmacoepidemiol Drug Saf 2012; 21:989-996.

11. Sawada $N$, Uchida $H$, Watanabe $K$, et al. How successful are physicians in eliciting the truth from their patients? A large-scale internet survey from patients' perspectives. J Clin Psychiatry 2012; 73:311-317.

12. Lindstrom E, Eriksson L, Levander S. Suicides during 7 years among a catchment area cohort of patients with psychoses. Nord J Psychiatry 2012; 66:8-13.

13. Misdrahi $D$, Petit $M$, Blanc $O$, et al. The influence of therapeutic alliance and insight on medication adherence in schizophrenia. Nord J Psychiatry 2012; $66: 49-54$.

14. Linnet $\mathrm{K}$, Halldorsson M, Thengilsdottir $\mathrm{G}$, et al. Primary nonadherence to prescribed medication in general practice: lack of influence of moderate increases in patient copayment. Fam Pract 2013; 30:69-75.

15. Montes JM, Medina E, Gomez-Beneyto M, Maurino J. A short message service

- (SMS)-based strategy for enhancing adherence to antipsychotic medication in schizophrenia. Psychiatry Res 2012; 200:89-95.

A study demonstrating the potential opportunities and challenges of adherence interventions using SMS. Three months of daily SMS reminders were used to prompt patients with schizophrenia to take their medication. Improvements in adherence were sustained at 6 months; however, approximately one-third of intervention patients were excluded because they did not receive the SMS messages.

16. Santana L, Fontenelle JM, Yucel M, Fontenelle LF. Rates and correlates of nonadherence to treatment in obsessive-compulsive disorder. J Psychiatr Pract 2013; 19:42-53.
17. Berger A, Edelsberg J, Sanders $\mathrm{KN}$, et al. Medication adherence and utiliza-

- tion in patients with schizophrenia or bipolar disorder receiving aripiprazole, quetiapine, or ziprasidone at hospital discharge: a retrospective cohort study. BMC Psychiatry 2012; 12:99.

This study demonstrated that patients may be at risk of nonadherence when discharged from hospital; medication possession ratios for patients with bipolar disorder or schizophrenia who initiated a new treatment on discharge were low (37.3\% and $55.1 \%$, respectively).

18. Scott J, Colom F, Pope M, et al. The prognostic role of perceived criticism, medication adherence and family knowledge in bipolar disorders. J Affect Disord 2012; 142:72-76.

19. Montreuil TC, Cassidy CM, Rabinovitch $M$, et al. Case manager-and patientrated alliance as a predictor of medication adherence in first-episode psychosis. J Clin Psychopharmacol 2012; 32:465-469.

20. Aggarwal NK, Sernyak MJ, Rosenheck RA. Prevalence of concomitant oral antipsychotic drug use among patients treated with long-acting, intramuscular, antipsychotic medications. J Clin Psychopharmacol 2012; 32:323-328.

21. Offord S, Lin J, Mirski D, Wong B. Impact of early nonadherence to oral

- antipsychotics on clinical and economic outcomes among patients with schizophrenia. Adv Ther 2013; 30:286-297.

This study used the prescription claims database to assess medication possession for patients with schizophrenia taking oral antipsychotic medications. Patients with medication refill gaps more than 30 days within first 3 months of prescription were at an increased risk of later discontinuation, demonstrating the importance of early adherence.

22. Waterreus A, Morgan VA, Castle D, et al. Medication for psychosis consumption and consequences: the second Australian national survey of psychosis. Aust N Z J Psychiatry 2012; 46:762-773.

23. Olivares JM, Alptekin $\mathrm{K}$, Azorin JM, et al. Psychiatrists' awareness of - adherence to antipsychotic medication in patients with schizophrenia: results from a survey conducted across Europe, the Middle East, and Africa. Patient Prefer Adherence 2013; 7:121-132.

A survey of 4722 psychiatrists across Europe, the Middle East and Africa, who estimated on average that $53 \%$ of their patients took less than $90 \%$ of their medication.

24. Alene M, Wiese MD, Angamo MT, et al. Adherence to medication for the

- treatment of psychosis: rates and risk factors in an Ethiopian population. BMC Clin Pharmacol 2012; 12:10.

This study reports that only $19.6 \%$ of a sample of 336 patients with schizophrenia and/or psychoses who were inpatients at a hospital in Ethiopia filled $100 \%$ of their prescriptions as prescribed.

25. Yang J, Ko YH, Paik JW, et al. Symptom severity and attitudes toward medication: impacts on adherence in outpatients with schizophrenia. Schizophr Res 2012; 134:226-231.

26. Vrijens $B$, De Geest $S$, Hughes DA, et al. A new taxonomy for describing and -1 defining adherence to medications. Br J Clin Pharmacol 2012; 73:691-705.

This study reports on a new taxonomy for describing adherence to medications, developed through expert consensus.

27. Lim SW, Kwon YS, Ha J, et al. Comparison of treatment adherence between selective serotonin reuptake inhibitors and moclobemide in patients with social anxiety disorder. Psychiatry Investig 2012; 9:73-79.

28. Jaeger $S$, Pfiffner $C$, Weiser $P$, et al. Adherence styles of schizophrenia patients identified by a latent class analysis of the Medication Adherence Rating Scale (MARS): a six-month follow-up study. Psychiatry Res 2012; 200:83-88.

29. Wong B, Mirski D, Lin J, Offord S. Among patients with schizophrenia nonadherence to antipsychotic medications early-on results in more hospitalizations and greater healthcare costs [Conference abstract]. Early Interv Psychiatry 2012; 6:99.

30. San L, Bernardo M, Gomez A, et al. Socio-demographic, clinical and treatment characteristics of relapsing schizophrenic patients. Nord J Psychiatry 2013; 67:22-29.

31. Witt K, van Dorn R, Fazel S. Risk factors for violence in psychosis: systematic

-1. review and meta-regression analysis of 110 studies. PLoS One 2013; 8:e55942.

A meta-analysis of factors associated with violent behaviour in adults with psychosis found that nonadherence was a risk factor for violence. The effects were heterogeneous, suggesting that this effect may be moderated by other factors (e.g. sample size, nonadherence measure).

32. Ugarte A, Martinez-Cengotitabengoa M, Alberich $S$, et al. Adherence and depression in bipolar patients with suicidal risk. Bipolar Disord 2012; 14:132.

33. Ruengorn $C$, Sanichwankul $K$, Niwatananun $W$, et al. A risk-scoring scheme

- for suicide attempts among patients with bipolar disorder in a Thai patient cohort. Psychol Res Behav Manag 2012; 5:37-45.

A case-control study comparing patients with major depressive disorder who attempted suicide and sought hospital treatment with matched controls; nonadherence to medications was retrospectively reported more often by patients who had attempted suicide or their families than controls.

34. Cullen BA, McGinty EE, Zhang Y, et al. Guideline-concordant antipsychotic - use and mortality in schizophrenia. Schizophr Bull 2012. [Epub ahead of print] A retrospective analysis of pharmacy claims for antipsychotic medication and mortality. Mortality reduced in patients with a medication possession ratio of more than $90 \%$. 
35. Simpson SH, Eurich DT, Majumdar SR, et al. A meta-analysis of the association between adherence to drug therapy and mortality. BMJ 2006; 333:15.

36. Loayza N, Crettol S, Riquier F, Eap CB. Adherence to antidepressant treatment: what the doctor thinks and what the patient says. Pharmacopsychiatry 2012; 45:204-207.

37. Stephenson JJ, Tunceli O, Gu T, et al. Adherence to oral second-generation

-1 antipsychotic medications in patients with schizophrenia and bipolar disorder: physicians' perceptions of adherence vs. pharmacy claims. Int J Clin Pract 2012; 66:565-573.

A study using novel linkage methodology. Researchers surveyed 153 physicians about 214 of their patients with schizophrenia or bipolar disorder, comparing patients' pharmacy refill records with physicians' estimates for individual patients. Physicians overestimated adherence for $67 \%$ of patients with bipolar disorder with low adherence and $94 \%$ of patients with schizophrenia with low adherence.

38. Thompson L, McCabe R. The effect of clinician-patient alliance and

- communication on treatment adherence in mental healthcare: a systematic review. BMC Psychiatry 2012; 12:87.

A systematic review identifying 17 of 23 studies reporting positive associations between physician-patient communication factors and adherence.

39. Quirk A, Chaplin R, Hamilton S, et al. Communication about adherence to

n. long-term antipsychotic prescribing: an observational study of psychiatric practice. Soc Psychiatry Psychiatr Epidemiol 2013; 48:639-647.

This study recorded and analysed consultations to provide insight into the dynamics of conversations about nonadherence. They found that nonadherence was often presented as a deliberate choice by the patient and responded to by their psychiatrist with a change of prescription.

40. Bowskill R, Clatworthy J, Parham R, et al. Patients' perceptions of information received about medication prescribed for bipolar disorder: implications for informed choice. J Affect Disord 2007; 100:253-257.

41. National Institute for Health and Clinical Excellence. Medicines adherence: involving patients in decisions about prescribed medicines and supporting adherence CG76. London: National Institute for Health and Clinical Excellence; 2009.

42. Coleman $\mathrm{Cl}$, Limone $\mathrm{B}$, Sobieraj $\mathrm{DM}$, et al. Dosing frequency and medication adherence in chronic disease [Review]. J Manage Care Pharm 2012; 18:527-539.

43. Medic G, Higashi K, Littlewood KJ, et al. Dosing frequency and adherence in chronic psychiatric disease: systematic review and meta-analysis. Neuropsychiatr Dis Treat 2013; 9:119-131.

44. Staring ABP, van der Gaag M, Duivenvoorden $\mathrm{HJ}$, et al. Why do patients with schizophrenia who have poor insight still take antipsychotics? Memory deficits as moderators between adherence belief and behavior. J Psychiatr Pract 2011; 17:320-329.

45. Gadkari A, McHorney C. Unintentional nonadherence to chronic prescription medications: how unintentional is it really? BMC Health Serv Res 2012; $12: 1-12$.

46. Seeman MV, Seeman N. The meaning of antipsychotic medication to patients with schizophrenia [Review]. J Psychiatr Pract 2012; 18:338-348.

47. Richardson M, McCabe R, Priebe S. Are attitudes towards medication adherence associated with medication adherence behaviours among patients with psychosis? A systematic review and meta analysis. Soc Psychiatry Psychiatr Epidemiol 2013; 48:649-657.

48. Jonsdottir $\mathrm{H}$, Opjordsmoen $\mathrm{S}$, Birkenaes $\mathrm{AB}$, et al. Predictors of medication

m. adherence in patients with schizophrenia and bipolar disorder. Acta Psychiatr Scand 2012; 127:23-33.

This study highlighted the role of substance use and insight in medication adherence for patients with bipolar disorder and schizophrenia.

49. Montes JM, Maurino J, de Dios C, Medina E. Suboptimal treatment adherence

- in bipolar disorder: impact on clinical outcomes and functioning. Patient Prefer Adherence 2013; 7:89-94.

A cross-sectional study of 303 primary care patients with bipolar disorder. Patients whose most recent episode was depressive had 3.41 times higher odds of suboptimal adherence, demonstrating a possible role for episode polarity in nonadherence.
50. Kronish IM, Edmondson D, Li Y, Cohen BE. Posttraumatic stress disorder and - medication adherence: results from the Mind Your Heart Study. J Psychiatr Res 2012; 46:1595-1599.

This study surveyed 535 patients who had recently experienced strokes or transient ischaemic attacks, and found that patients with possible PTSD also reported nonadherence.

51. Edmondson D, Horowitz CR, Goldfinger JZ, et al. Concerns about medications mediate the association of posttraumatic stress disorder with adherence to medication in stroke survivors. Br J Health Psychol 2013. [Epub ahead of print]

52. Rao D, Feldman BJ, Fredericksen RJ, et al. A structural equation model of HIV-related stigma, depressive symptoms, and medication adherence. AIDS Behav 2012; 16:711-716.

53. Sajatovic M, Levin J, Tatsuoka $C$, et al. Six-month outcomes of customized adherence enhancement (CAE) therapy in bipolar disorder. Bipolar Disord 2012; 14:291-300.

54. Sajatovic M, Levin J, Tatsuoka C, et al. Customized adherence enhancement for individuals with bipolar disorder receiving antipsychotic therapy. Psychiatr Serv 2012; 63:176-178.

55. Gleeson JF, Cotton SM, Alvarez-Jimenez M, et al. A randomized controlled trial of relapse prevention therapy for first-episode psychosis patients: outcome at 30-month follow-up. Schizophr Bull 2013; 39:436-448.

56. Petry NM, Rash CJ, Byrne S, et al. Financial reinforcers for improving medication adherence: findings from a meta-analysis. Am J Med 2012; 125:888-896

57. Stein BD, Kogan JN, Mihalyo MJ, et al. Use of a computerized medication

- shared decision making tool in community mental health settings: impact on psychotropic medication adherence. Community Ment Health J 2013; 49:185-192.

This study reported on the use of a preconsultation computer questionnaire that sought to elicit patients' perspectives on their medication. No evidence was reported for improvements in adherence at 180 days of follow-up.

58. Velligan D, Mintz J, Maples $\mathrm{N}$, et al. A randomized trial comparing in person

- and electronic interventions for improving adherence to oral medications in schizophrenia. Schizophr Bull 2012. [Epub ahead of print]

This study highlighted the potential efficiency of using electronic monitoring to support adherence. Researchers compared patients receiving electronic monitoring with patients receiving home visits and found that both improved adherence relative to treatment as usual.

59. Zimmerman N. Telemonitoring of medication adherence in patients with major mental illness: watching the patient as well as the pills. Stud Health Technol Inform 2012; 182:189-196.

60. Read U. 'I want the one that will heal me completely so it won't come back

- again': the limits of antipsychotic medication in rural Ghana. Transcult Psychiatry 2012; 49:438-460.

Qualitative case studies describing the experiences of antipsychotic treatment of Ghanaian patients and their families, highlighting the importance of feelings of physical weakness, side effects and spiritual beliefs in nonadherence.

61. Horne R, Graupner LD, Frost S, et al. Medicine in a multicultural society: the effect of cultural background on beliefs about medications. Soc Sci Med $2004 ; 59: 1307-1313$

62. Kopelowicz A, Zarate R, Wallace CJ, et al. The ability of multifamily groups

n. to improve treatment adherence in Mexican Americans with schizophrenia. Arch Gen Psychiatry 2012; 69:265-273.

This study reports a randomized controlled trial of multifamily group therapy for medication adherence for Mexican-Americans with schizophrenia. Significant improvements in adherence and hospitalization were shown at 12 months after intervention end. This study demonstrates how interventions can be culturally adapted through being responsive to the needs of patients.

63. Michie S, Richardson M, Johnston $\mathrm{M}$, et al. The behavior change technique

- taxonomy ( $v 1)$ of 93 hierarchically clustered techniques: building an international consensus for the reporting of behavior change interventions. Ann Behav Med 2013. [Epub ahead of print]

This study reports on a taxonomy of methods for changing behaviour. It acts as a potential resource for describing the contents of adherence interventions. 